\title{
Grupos de Orientação Parental: um relato de experiência
}

\section{Parental Guidance Groups: an experience report}

\section{Julio Cezar Goncalves' 1 (1) Josiane da Silva Delvan ${ }^{2}$ (1) Andressa Juliana de Oliveira ${ }^{3}$ (1) Ariadny Suci Abbud 4 (1) Victória Niebuhr $\operatorname{Loos}^{5}$ (1) Mariana de Oliveira Bortolatto ${ }^{6}$ (1)}

Autor para correspondência. Universidade do Vale do Itajaí (Itajaí). Santa Catarina, Brasil. psico.julio@edu.univali.br 2-4Universidade do Vale do Itajaí (Itajaí). Santa Catarina, Brasil. josidelvan@univali.br, andressajuliana.psi@gmail.com, ariadnypsicologa@gmail.com 5-6Universidade Federal de Santa Catarina (Florianópolis) Santa Catarina, Brasil.victorialoos@hotmail.com, mariana_bortolatto@hotmail.com

RESUMO | A conjuntura familiar é uma das maiores influências no processo de desenvolvimento infantil, de modo que, estudos voltados às práticas parentais possuem significativa relevância científica, acadêmica e social. Dessa forma, o presente artigo objetiva relatar a experiência da realização de Grupos de Orientação Parental no período de três anos em uma cidade de Santa Catarina. Trata-se de um estudo Descritivo e Transversal do tipo Relato de Experiência. Participaram 49 pais, que foram seccionados em seis grupos no decurso dos três anos de aplicação, com encontros semanais. Por conseguinte, ao sobrepor Práticas Grupais com Orientação para Pais, evidenciou-se que os benefícios dos atendimentos em grupo são diversos, considerando que este formato favorece oportunidades para aprendizagem, maior apoio entre os membros, redução do estigma e aumento do autofoco. Contudo, os desafios aos terapeutas são muitos e o desenvolvimento de determinadas características profissionais necessárias para o alcance dos resultados esperados. Essa asserção caracteriza notadamente a importância dos Estágios Obrigatórios, podendo ser uma importante oportunidade para a aquisição dessas competências, na mesma medida que possibilita aplicar a teoria na prática.

PALAVRAS-CHAVE: Orientação Parental. Grupos de Orientação. Família.

\begin{abstract}
The family is one of the biggest influences on the child development process, so that studies focused on parenting practices have scientific, academic and social generation. Thus, the article aims to report the experience of conducting Parental Guidance Groups over a threeyear period in a city on Santa Catarina state, Brazil. This is a Descriptive and Cross-sectional study of the Experience Report type. 49 parents participated, who were divided into six groups during the three years of application, with weekly meetings. Therefore, when superimposing Group Practices with Parent Orientation, it became evident that the benefits of group care are diverse, considering that this format favors opportunities for learning, greater support among members, reducing stigma and increasing autofocus. However, the challenges for therapists are many and the development of certain characteristics is necessary to achieve the expected results. This assertion notably characterizes the importance of Mandatory Internships, and can be an important opportunity for the acquisition of these competences, in the same measure that makes it possible to take an important step in the process: applying the theory in practice.
\end{abstract}

KEYWORDS: Parental Guidance. Orientation Groups. Family. 


\section{Introdução}

Considerada uma das principais atividades grupais humanas, a conjuntura familiar se instituiu como uma fonte primordial que garantiu a sobrevivência e a adaptação da espécie humana ao longo dos milênios, estendendo-se à contemporaneidade. Paralelo a esta conjuntura, e amparado por múltiplas pesquisas, os estudos são consensuais ao denotar a influência de aspectos multifatoriais no decurso do processo de Desenvolvimento Humano (DH) e a família se mantêm no posto como um dos fatores fundamentais (Teixeira, Bardagi \& Gomes, 2004; Pereira et al., 2018; Weber et al., 2004; Macarini et al., 2010).

Outrossim, para que a compreensão sobre a influência e importância da conjuntura familiar se tornasse alcançável no nível prático, diversos teóricos recorreram ao longo dos anos a abordagens sistemáticas e interdisciplinares, o que resultou na concepção de dois modelos comumente utilizados para investigar fenômenos concernentes ao $\mathrm{DH}$, e consequentemente da conjuntura familiar: o Modelo Bioecológico do Desenvolvimento Humano e a Teoria de Investimento Parental (Bronfenbrenner, 2002, Leme et al., 2016).

Segundo o Modelo Bioecológico do Desenvolvimento Humano (MBDH), o indivíduo está inserido em sistemas concêntricos, ou seja, contextos em que o processo de desenvolvimento é o produto resultante da interação entre o indivíduo e o ambiente em que se insere, desde o ambiente familiar, o ambiente escolar, a vizinhança, até o local de trabalho dos pais, os sistemas políticos, religiosos e ideológicos. De modo geral, o modelo postula que o indivíduo é concebido a partir de processamentos interacionais de si próprio com esses múltiplos ambientes, que são compostos por outras pessoas, símbolos, objetos, se adaptando e se modificando em um continuum no espaço-tempo (Bronfenbrenner, 2002; Benetti et al., 2013). Já a Teoria de Investimento Parental, enquanto modelo embasado em uma perspectiva evolucionista, propõe que as habilidades referentes às estratégias parentais da espécie humana foram selecionadas no decorrer do período evolutivo por propiciar uma maior possibilidade de adaptação e sobrevivência. Dessa forma, o sistema familiar é regido por práticas dispensadas pelos pais aos filhos: comunicação, apoio emocional, cuidado, proteção, afeto, entre outras, como garantias de sobrevivência da prole (Manfroi, Macarini \& Vieira, 2011; Bossardi \& Vieira, 2011).
A partir desses movimentos, indagações sobre quais as formas mais efetivas para educar os filhos, se instituírem como pautas das pesquisas científicas desde o início do século XX, de modo que vários conceitos foram desenvolvidos com o objetivo de categorizar e organizar os achados científicos sobre a família, dentre eles os conceitos de parentalidade, práticas educativas parentais e estilos parentais (Ribeiro, Gomes \& Felizardo, 2015; Macarini et al., 2010)

O conceito de parentalidade é mais geral, e pode ser entendido como o resultado das práticas educativas parentais aplicadas e do estilo parental adotado. É caracterizada, principalmente, pela relação entre duas gerações em que os pais têm o desígnio de prover para o filho o cuidado, a proteção, a educação e a integração com outras redes familiares ou institucionais, com objetivo final de constituir infâncias adaptativas, saudáveis e promissoras na adultez (Bossardi \& Vieira, 2011; Gaspar \& Matos, 2016; Hoghughi, 2004; Manfroi; Macarini \& Vieira, 2011; Ribeiro; Gomes \& Felizardo, 2015).

Já as práticas educativas parentais constituem-se em estratégias utilizadas pelos pais para alcançarem finalidades em distintas esferas como a escola e os meios sociais, e, são caracterizados, pelo conjunto de comportamentos e valores apresentados pelos pais que atuam na regência e em grande parte na consolidação da identidade da criança. Usualmente, as práticas educativas parentais são as formas com que pais educam e ensinam os seus filhos acerca do mundo, e estas podem envolver múltiplas categorias: monitoria positiva, comportamento moral, disciplina relaxada, monitoria negativa, abuso físico punição inconsistente e negligência. A monitoria positiva consiste no estabelecimento de regras, supervisão dos pais em relação as atividades do filho e manutenção de uma comunicação assertiva e empática. O comportamento moral diz respeito a adaptação às regras do grupo e o quanto os pais educam seus filhos com base nas mesmas. A disciplina relaxada é a falta de regras e punições inconsistentes. A monitoria negativa se caracteriza pelas instruções descontextualizadas das consequências e manutenção de um ambiente de convivência hostil. Já o abuso físico e a negligência são a ausência de atenção e afeto, violência física e psicológica e abandono do filho (Hoghughi, 2004; Mondin, 2008). 
Os estilos parentais são, basicamente, uma extensão das práticas educativas parentais, e se traduzem na maneira com que os pais se apresentam a nível de atitudes e instruções na execução dessas práticas. Essencialmente, é o clima emocional em que decorrem as relações entre ambos, traduzidos no tom e altura de voz, a linguagem corporal, instruções acerca das maneiras de perceber e agir no mundo e as mudanças de humor. São classificadas em quatro categorias com base no cuidado e em atitudes compreensivas, caracterizados por: pais autoritários, que usam de coerção como forma disciplinar; pais autoritativos, que não usam disciplina coerciva e partem do princípio da comunicação assertiva; pais negligentes, que não exercem o papel de pai e decaem progressivamente na manutenção da relação pai e filho; pais indulgentes, se caracterizam por manter práticas voltadas para reforçar quaisquer comportamentos dos filhos, sem imposição de limites ou regras (Ribeiro, Gomes \& Felizardo, 2015).

As práticas educativas parentais, assim como os estilos parentais, podem se configurar como fatores de risco no desenvolvimento infantil (Patias, Siqueira \& Dias, 2004). Dentre esses múltiplos fatores, um dos aspectos primordiais e indispensáveis a ser intensamente discutido é sobre a prevenção da violência infantil. Apesar das diversificadas políticas públicas e atuações dos órgãos de proteção voltados ao público infantil, estimativas acerca dos maus-tratos e abusos possuem índices altos e apontam que $22 \%$ dos adultos no mundo sofreram abusos físicos na infância, $36 \%$ sofreram abuso emocional e $16 \%$ sofreram negligência, não havendo significativas diferenças entre gêneros (Gonçalves \& Vieira, 2018).

Ainda, estudos realizados nas últimas décadas no Brasil, também evidenciaram consequências negativas advindas das práticas parentais coercivas, em que mantêm correlação positiva no que se refere a vida adulta com uma maior prevalência na transgressão das normas sociais, menos apoio social, menor capacidade de resiliência, baixa autoestima e baixa tolerância à frustação (Brasil, 2005; Weinfurter, 2015). Outros estudos realizados confirmam a tese de que as crianças manifestam maior sofrimento emocional e dificuldade de controlar suas emoções quando expostas às expressões emocionais negativas e manutenção de práticas coercivas por parte dos pais, verificando-se resultados positivos quando implementado orientação para os pais sobre o uso da teoria do reforçamento positivo para manutenção de comportamentos adaptativos (Mondin, 2008).

A nível interventivo, uma revisão sistemática realizada recentemente sobre Grupos de Orientação Parental para pais no Brasil obteve como resultado o reduzido número de cinco artigos sobre o tema entre 2014 e 2018. Na síntese qualitativa, os autores ressaltam que a legitimidade dos estudos acerca da validade interna e externa abrangeram somente dois estudos, estes com uso de pré-teste e pós-teste. Um dos estudos fez uso de questionários e dois estudos não realizaram nenhum processo de avaliação. Em relação ao número da amostra, um estudo contou com uma amostra máxima de 97 participantes, outro contou com uma amostra média de 22 participantes e os estudos restantes com uma amostra abaixo de dez participantes (Gonçalves, Oliveira \& Delvan, 2018).

Não obstante, apesar da revisão supracitada ter denotado dois estudos quase-experimentais com decorrências positivas quanto à efetividade, de maneira geral, esses resultados ainda são incipientes, com o adendo de que os restantes dos estudos não foram experimentais, o que prediz a necessidade de pesquisas com maior rigor empírico com vistas a efetividade da prática de grupos de orientação parental (Neufeld, Godoi \& Propais, 2014).

Porquanto, ao sobrepor as práticas de orientação parental às teorias de práticas grupais atuais, estas são possíveis por deterem a característica, principalmente, da homogeneidade. Ao mesmo tempo, os encontros são estruturados e baseados em protocolos e cronogramas preestabelecidos, mas que, inversamente, a partir das demandas contextuais do grupo, o encontro pode ser conduzido de modo dinâmico e flexível mesmo vinculado ao protocolo, visando a adaptação eficaz do grupo (Neufeld et al., 2017). Os encontros contam com o estabelecimento de um contrato com critérios bem estabelecidos já nas reuniões iniciais, em que se alude a permanência dos participantes, bem como, os procedimentos de pré-teste e pós-teste. Essas reuniões iniciais partem do princípio da firmação do rapport entre as compartes, geralmente sendo reforçado através de dinâmicas. Os encontros incluem tarefas, sendo que estas devem ser revisadas no encontro seguinte, a fim de consolidar e avaliar a aprendizagem, assim como, incentivar o engajamento nos exercícios propostos (Neufeld et al., 2017). 
Em se tratando de práticas grupais com conteúdos relacionados a parentalidade e desenvolvimento infantil, vale destacar o Programa ACT Para Educar Filhos em Ambientes Seguros, protocolo interventivo elaborado pela American Psychology Association (APA). O Programa tem se enquadrado tanto no nível de promoção dos fatores terapêuticos citados como também no nível de prevenção à violência infantil, já que seu principal objetivo é incorporar métodos interventivos para prevenir antecipadamente a violência infantil e inserir na comunidade a perspectiva de proteção à criança e habilitar famílias a criarem ambientes seguros, estáveis e saudáveis no decorrer no desenvolvimento infantil (Weymouth \& Howe, 2011; APA, 2009).

O Programa, denominado ACT, teve auxílio da National Association for the Education of Young Children (NAEYC), uma associação sem fins lucrativos nos Estados Unidos que representa professores de educação infantil, paraeducadores, diretores de centro, treinadores, educadores de faculdades, famílias de crianças pequenas, formuladores de políticas e defensores e baseou-se em resultados experimentais de prevenção à violência na infância. O ACT versa e auxilia os profissionais e as famílias a deliberarem sobre os comportamentos infantis apropriados a cada etapa do desenvolvimento, dando ênfase à importância de os pais desempenharem um papel positivo para a criança no sentido de monitorar e conduzir o comportamento por meio de práticas aversivas em detrimento à violência (Silva et al., 2016).

Face ao exposto, é possível destacar as fortes influências que o ambiente e as práticas parentais exercem no desenvolvimento humano, desde consequências cognitivo-comportamentais, psicossociais até consequências de ordem cultural, o que destaca a relevância social, científica, acadêmica e de saúde de que estudos sobre práticas parentais sejam conduzidos, visando a promoção da qualidade de vida das famílias. Ainda, ressalta-se a importância de modelos interventivos voltados à orientação de práticas parentais positivas, modelos estes com o objetivo de propiciar aos pais a capacidade de oferecerem um processo de desenvolvimento/aprendizagem de qualidade às crianças, de modo que as auxiliem a inserirem-se nos ambientes e contextos de modo assertivo, por meio de interações positivas e visando uma vida adulta promissora. Haja vista que muitos pais carecem de habilidades para manter práticas educativas mínimas, as práticas grupais com enfoque na orientação parental, abalizada por pesquisas baseadas em evidências, se enquadram como métodos efetivos para a ocorrência de novos aprendizados acerca do modo adequado de educar os filhos (APA, 2009).

Assim, este artigo tem como objetivo relatar a experiência da aplicação de Grupos de Orientação Parental no período de três anos em uma cidade do litoral de Santa Catarina, descrevendo os encontros realizados e as temáticas abordadas, bem como os resultados das intervenções.

\section{Método}

Trata-se de um relato de experiência da aplicação de Grupos de Orientação Parental, no período de 2015 a 2018. Os Grupos de Orientação Parental ocorreram ao longo de três anos e contaram com a participação de 49 pais, com encontros semanais distribuídos conforme disponibilidade de horários dos participantes, de acordo com o Programa ACT.

\section{Resultados}

\section{Descrição dos Encontros realizados com os Grupos de Pais}

O primeiro encontro realizado com os pais foi destinado à Compreensão do Comportamento das Crianças, em que se abordou especificamente sobre o processo de desenvolvimento humano, perpassando as diferenças físicas, encefálicas, cognitivo-comportamentais e psicossociais na medida em que a criança cresce. Concomitante à descrição das diferenças mais proeminentes, inseriu-se marcos conceituais acerca do processo de aprendizagem humana e, consequentemente, de que maneira o modo das crianças se comportarem nos ambientes está inteiramente ligada ao seu processo de desenvolvimento e processo de aprendizagem (Martins, 2014). Discorreu-se pontualmente sobre o impacto que os primeiros anos de vida tem no desenvolvimento infantil, já que são as primeiras interações que estas se fixam para diversas outras respostas que darão ao ambiente (Bronfenbrenner, 2002). Deu-se destaque que as práticas educativas parentais podem se configurar como fatores de risco no desenvolvimento infantil (Patias, Siqueira \& Dias, 2004; Brasil, 2005; 
Weinfurter, 2015), assim como, discorreu-se sobre a importância da mediação dos pais no decurso do processo de aprendizagem, no qual são cruciais para a orientação, modelagem e modelação no que concerne a expressão de comportamentos adaptativos (Caleiro \& Silva, 2012).

Imbuídos dos aspectos gerais sobre desenvolvimento infantil, aprendizagem humana e papel dos pais como mediadores de tais processos, o segundo encontro foi destinado à psicoeducação acerca dos impactos da Exposição da Criança à Violência. Deu-se ênfase na descrição de quais formas as crianças podem ser expostas à violência e como isto pode prejudicá-las. Há três maneiras em que as crianças podem ser expostas à violência, como vítimas, como agressores e como testemunhas, em que estas podem ocorrer através de abusos físicos, verbais, sexuais e através da negligência. Tendo em vista que os primeiros anos de vida são um período crucial no desenvolvimento infantil, a exposição a qualquer forma de violência pode trazer consequências prejudiciais às crianças (Goulart et al., 2015).

A violência como prática educativa é uma violação do direito da criança na mesma medida em que aumenta o risco de que a criança envolvida nela sofra sérias consequências, inclusive o óbito (Martins, 2010; Rates, 2015). Nos últimos anos, múltiplos estudos têm descrito a reverberação dos conflitos conjugais no desenvolvimento infantil, e, apesar das diferenças no nível de consequências de uma criança para outra, há evidências consistentes denotando fortes correlações entre conflitos conjugais e problemas de comportamento, em distintas faixas etárias (Benetti, 2006; Goulart et al., 2015). De modo subjacente, os conflitos conjugais presenciados pela criança, além de se caracterizarem como potenciais fatores de risco ao longo do processo de desenvolvimento se apresentam como modelos violentos de aprendizagem (Weinfurter, 2015).

O terceiro encontro foi sobre o tema Entendendo e Controlando a Raiva, voltada especificamente aos pais. Primeiramente, foi elaborado um fluxograma de casos com situações em que as crianças fazem os pais sentirem raiva e como, frequentemente, esses pais lidam com isto. A partir desses dados, foi realizada a psicoeducação acerca do modelo cognitivo, ou seja, de que modo se dá a relação entre pensamentos, emoções e comportamentos. No fluxograma de casos, foi discorrido sobre qual a maneira adequada para controlar a raiva, e como esta envolve, primariamente, a identificação de pensamentos ao lidar com situações em que os filhos as deixam com raiva, e assim, buscar não agir impulsivamente. De acordo com Knapp \& Beck (2008), existe uma inter-relação entre cognição, emoção e comportamento. Uma determinada situação pode ativar alguns pensamentos no indivíduo, o que gera emoções e comportamentos. Esta relação é importante para compreender a raiva pois, na maioria das vezes, a sensibilidade em relação à situação ocorre justamente pela interpretação cognitiva desta. Ao ter conhecimento disto, os pais podem identificar quais pensamentos ocorrem ao vivenciar determinadas situações com seus filhos, examinar as evidências da realidade e, com isto, corrigir quaisquer conteúdos distorcidos em tais pensamentos, resultando em comportamentos mais funcionais para com seus filhos (Neufeld \& Rangé, 2017).

O relaxamento progressivo foi utilizado para reduzir as atividades do sistema simpático e propiciar a sensação de relaxamento, e, concomitante ao escaneamento corporal, permitiu a preparação para realização da exposição via imaginação. O escaneamento corporal é uma atividade frequentemente utilizada em treinamentos de mindfulness e consiste em manter a atenção plena e aberta, no momento presente, para os membros do corpo de forma progressiva (Germer, Siegel \& Fulton, 2016). Essa atividade busca ampliar o nível de consciência das percepções dos sentidos, pensamentos e sentimentos, e auxilia na redução do estresse (Neufeld \& Rangé, 2017).

Com base nas premissas do modelo cognitivo explanado no encontro anterior, o quarto encontro foi sobre "Como Ensinar as Crianças a Lidar com a Raiva". As crianças, do mesmo modo que os adultos, sentem raiva a partir de três ordens básicas: biológicas, ontogenéticas (aprendidas) e socioculturais. As crianças apresentam sentimentos como a raiva por questões adaptativas, e é tarefa dos pais ensinarem seus filhos a controlar tais sentimentos. Assim, para auxiliar as crianças a lidar com a raiva, é importante controlar a própria raiva e, acima de tudo, evitar a violência. 
Essa atitude pode gerar dois resultados importantes e essenciais: a aprendizagem via modelação de como controlar os sentimentos e desenvolvimento de estratégias educativas assertivas (Barbato, 2010).

Embora muitas vezes os pais ainda se espelham na educação recebida, normalmente severa e baseada no conceito de "certo e o errado", este estilo de prática parental não se aplica à família contemporânea, devido às complexidades apresentadas pela mesma. O castigo físico, em sua quase totalidade, não produz os efeitos esperados e pode abalar a relação de pai e filho, prejudicar a autoestima e acarretar sérios riscos à saúde física e psicológica da criança (Ribeiro, 2007). Não obstante, as crianças têm conflitos com adultos e com as outras crianças, pois estão em estágios desenvolvimentais diferentes e suas reações não têm o propósito de incomodar os pais, mas são comportamentos necessários para que as crianças se desenvolvam e se adaptem ao ambiente. Crianças que obtém modelos internos seguros possuem mais chances de apresentar um maior conhecimento das próprias emoções, contudo, para isto, é preciso que os pais possuam práticas positivas, compartilhando sentimentos, suas causas e consequências, estes condizentes aos princípios da modelagem e da modelação (Martins, 2010). Práticas parentais positivas contribuem para o funcionamento social e emocional das crianças, o que demonstra a importância de os pais e/ou cuidadores engajarem-se em observar e orientar as interações sociais dos filhos, bem como suas expressões emocionais (Rebelo, 2012).

Um dos temas de maior interesse e dúvidas se deu no quinto encontro, que tratou sobre o Impacto das Tecnologias na Vida das Crianças. Essas dúvidas corroboram com os questionamentos que a sociedade tem feito assim como pesquisadores da área de desenvolvimento humano, tendo em vista a período prolongado que crianças e jovens mantem-se frente às Tecnologias de Informação e Comunicação (vídeo games, computadores, televisão, celulares, tablets). Contemporaneamente, as crianças são inseridas em um mundo alicerçado por relações sociais, mantidas via redes computacionais, assim como processos de aprendizagem informatizados, ausentando-se de contatos físicos com as pessoas e ambiente (Paiva \& Costa 2015; Barbosa et al., 2014; Momo, 2014). Todavia, esse modelo de intercâmbio possui múltiplas implicações no processo de desenvolvimento humano para além dos aspectos psicossociais e psicomotores, corroborando com um estudo voltado especificamente para a relação de crianças com desenhos animados realizado no Brasil por Miguel e Contino (2013), em que evidenciaram uma elevada alusão das crianças aos personagens e seus comportamentos, muitas vezes através de imitações fidedignas e violentas, resultados que favorecem o cuidado e monitoramento que os pais, como responsáveis, devem ter com os conteúdos e períodos que os filhos consomem através dos meios eletrônicos.

Como aprendemos a nos comportar como pais, quais são os estilos parentais mais comuns e suas implicações na vida dos filhos, foram os temas do sexto encontro. Os estilos parentais podem ser classificados em quatro categorias com base no cuidado e em atitudes compreensivas, caracterizados por: pais autoritários, que usam de coerção como forma disciplinar; pais autoritativos, que não usam disciplina coerciva e partem do princípio de comunicação assertiva; pais negligentes, que não exercem o papel de pai e decaem progressivamente na manutenção da relação pai e filho; pais indulgentes, se caracterizam por manter práticas voltadas para reforçar quaisquer comportamentos dos filhos, sem imposição de limites ou regras. Os estilos parentais são indicadores que possibilitam aferir a qualidade das práticas educativas parentais no seio de uma família, tendo em vista que, dependendo das práticas educativas parentais expressas, define-se o estilo parental conexo a cada pai ou mãe (Ribeiro, Gomes \& Felizardo, 2015).

Para auxiliar os participantes, optou-se por elaborar potenciais estratégias educativas que os auxiliassem a modificar seus estilos parentais, tendo em vista que em sua maioria, e vinculados à cultura do "certo" ou "errado", o estilo parental mais frequente foi o autoritário, com uso de práticas coercitivas como meio disciplinar. Foi intensamente reforçado que, de modo geral, as crianças pequenas se comportam mal porque estão aprendendo as coisas, e que é importante que os pais entendam e aceitem as crianças como elas são, não esperando delas que se comportem como adultas (Caleiro \& Silva, 2012). Ainda, de acordo com Pasquali et al. (2012), as pesquisas mostram que os estilos parentais estão relacionados a diversos aspectos do desenvolvimento psicossocial de crianças e adolescentes, como, por exemplo, autoestima, ajustamento social, psicopatologia e desempenho escolar, sendo ainda relacionados aos estímulos nos filhos para a disciplina, a independência, a autonomia e a responsabilidade, ao mesmo tempo em que modelam os comportamentos considerados adequados socialmente pelos pais. 
O sétimo encontro voltou-se a importância de os pais prestarem atenção nos comportamentos e atitudes dos filhos em todas suas interações, ou seja, como manter a Disciplina para Comportamentos Positivos. Foi utilizado o role play como meio de evidenciar aos pais o quanto, em sua grande maioria, se dá mais atenção aos comportamentos e situações de conflito do que dos momentos em que as crianças estão se comportando adequadamente (Ribeiro et al., 2007). Tendo em vista que a punição física é um comportamento ainda intrínseco na cultura ocidental, pois esta geralmente é contextualizada como prática educativa, conceito passado de geração para geração, auxiliando na construção do estilo parental de boa parte dos pais (Cecconello, Antoni \& Kolller, 2003), a psicoeducação sobre as diferenças entre punição e disciplina tornam-se importante para evitar que a punição continue sendo vista como prática educativa. Cabe a família ensinar a criança quais os comportamentos adequados que devem ser apresentados e saber como valorizá-los. Segundo Pasquali et al. (2012) e Benchaya et al. (2011) a família é considerada o principal apoio ao indivíduo na infância. É no ambiente familiar que as crianças constroem inicialmente suas crenças, valores, regras, atitudes, cultura, incluindo os comportamentos que são considerados adequados nesta cultura.

Por fim, na figura 1 é possível observar o delineamento dos encontros.

Figura 1. Delineamento dos encontros, distribuídos a partir das temáticas.

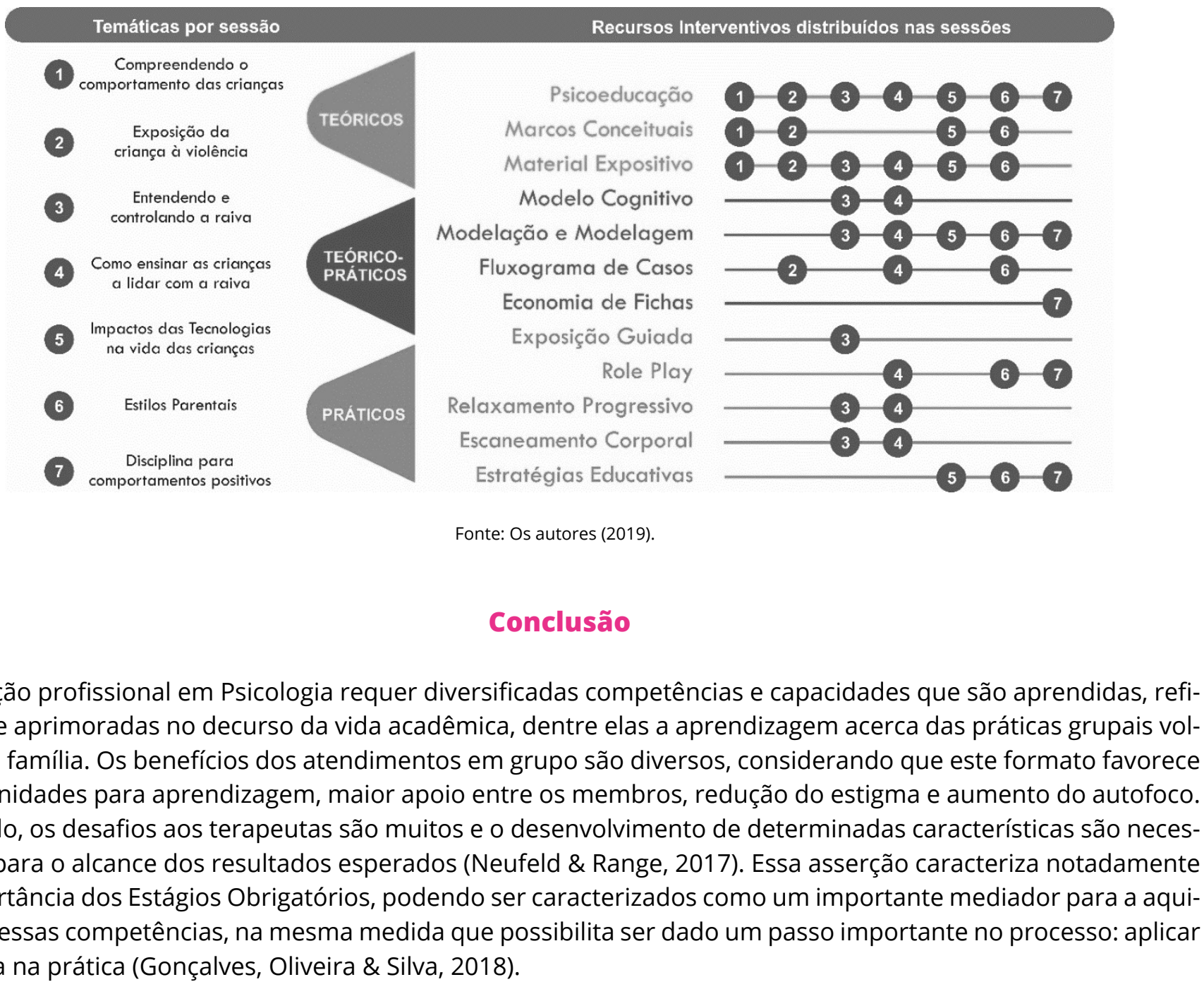

A atuação profissional em Psicologia requer diversificadas competências e capacidades que são aprendidas, refinadas e aprimoradas no decurso da vida acadêmica, dentre elas a aprendizagem acerca das práticas grupais voltadas à família. Os benefícios dos atendimentos em grupo são diversos, considerando que este formato favorece oportunidades para aprendizagem, maior apoio entre os membros, redução do estigma e aumento do autofoco. Contudo, os desafios aos terapeutas são muitos e o desenvolvimento de determinadas características são necessárias para o alcance dos resultados esperados (Neufeld \& Range, 2017). Essa asserção caracteriza notadamente a importância dos Estágios Obrigatórios, podendo ser caracterizados como um importante mediador para a aquisição dessas competências, na mesma medida que possibilita ser dado um passo importante no processo: aplicar a teoria na prática (Gonçalves, Oliveira \& Silva, 2018). 
Uma das limitações observadas, é de que o Programa ACT Para Educar Filhos em Ambientes Seguros, por ter sido elaborado e efetivado com amostras americanas, possui seu foco na prevenção da violência infantil e com tarefas, muitas vezes, de difícil aquisição pelos participantes brasileiros, notadamente relacionado ao nível de escolarização. Essa diferença exigiu dos facilitadores uma adaptação na forma de apresentar os temas, na mesma medida em que necessitavam aprender a dominar técnicas além do relato verbal, para auxiliar na participação dos pais pouco verbais.

Conquanto, apesar de o presente estudo ser um relato de experiência, ressalta-se que o levantamento de informações com os pais participantes dos grupospermitiu observar dados pertinentes quanto à ciência parental. Ademais, conclui-se que os Grupos de Orientação Parental foram uma experiência importante para preparação à prática profissional. De maneira subjacente, a partir da observação de três anos de aplicação, assinala-se a necessidade de programas elaborados a partir de amostras brasileiras, para que as intervenções sejam organizadas a partir das reais necessidades do público-alvo em questão, e que, transversalmente, se eleve os estudos experimentais com objetivo de que orientações voltadas à pais e/ou cuidadores, possam efetivamente auxiliálos na tarefa de serem pais.

\section{Contribuições dos autores}

Gonçalves JC Participou das revisões da literatura sobre o tema, condução dos grupos de orientação parental, avaliação das intervenções, redação do artigo científico. Delvan JSA Participou da orientação acerca das revisões da literatura sobre o tema, supervisão dos da condução dos grupos, orientação sobre avaliação das intervenções e correção final do artigo científico. Oliveira AJ, Abbud AS, Loos VN e Bortolatto MO participaram das revisões da literatura sobre o tema, condução dos grupos de orientação parental e avaliação das intervenções.

\section{Conflitos de interesses}

Nenhum conflito financeiro, legal ou político envolvendo terceiros (governo, empresas e fundações privadas, etc.) foi declarado para nenhum aspecto do trabalho submetido (incluindo, mas não se limitando a subvenções e financiamentos, participação em conselho consultivo, desenho de estudo, preparação de manuscrito, análise estatística, etc.).

\section{Referências}

American Psychological Association. (2009). Programa ACT para Educar Crianças em Ambientes Seguros. https://www.apa. org/act

Barbato, R. G., Correa, A. K., \& Souza, M. C. B. M. (2010). Aprender em grupo: experiência de estudantes de enfermagem e implicações para a formação profissional. Revista de Enfermagem, 14(1), 48-55. https://doi.org/10.1590/S141481452010000100008

Barbosa, G. C., Ferreira, M. M. G. A., Borges, L. M. \& Santos, A. G. (2014, agosto). Tecnologia digitais: possibilidades e desafios na educação infantil. ESUD - XI Congresso Brasileiro de Ensino Superior a Distância, Florianópolis, SC, Brasil. https://www.academia.edu/15830408/ TECNOLOGIAS_DIGITAIS_POSSIBILIDADES_E_DESAFIOS_ NA_EDUCA\%C3\%87\%C3\%830_INFANTIL

Beck, J. G., \& Coffey, S. F. (2005). Group cognitive behavioral treatment for PTSD: treatment of motor vehicle accident survivors. Cognitive and Behavioral Practice, 12(3), 267-277. https://doi.org/10.1016/s1077-7229(05)80049-5

Benchaya, M. C., Bisch, N. K., Moreira, T. C., Ferigolo, M. \& Barros, H. M. T. (2011). Pais não autoritativos e o impacto no uso de drogas: a percepção dos filhos adolescentes. Jornal da Pediatria, 87(3), 238-244. https://doi.org/10.1590/S002175572011000300010

Benetti, I. C., Vieira, M. L., Crepaldi, M. A., \& Schneider, D. R. (2013). Fundamentos da teoria bioecológica de Urie Bronfenbrenner. Pensando Psicologia, 9(16), 89-99. https:// doi.org/10.16925/pe.v9i16.620

Benetti, S. P. C. (2006). Conflito Conjugal: impacto no desenvolvimento psicológico da criança e do adolescente. Psicologia: Reflexão e Crítica, 19(2), 261-268. https://doi. org/10.1590/S0102-79722006000200012

Borsa, J. C., \& Nunes, M. L. T. (2011). Aspectos psicossociais da parentalidade: O papel de homens e mulheres na família nuclear. Psicologia e Argumentos, 29(64), 31-39. https:// periodicos.pucpr.br/index.php/psicologiaargumento/ article/view/19835

Bossardi, C. N., \& Vieira, M. L. (2011). Cuidado paterno e desenvolvimento infantil. Revista de Ciências Humanas, 44(1), 205-221. https://doi.org/10.5007/21784582.2010v44n1p205

Brasil. Secretaria de Atenção a Saúde.(2005). Violência Doméstica contra criança e adolescente. Fundo das Nações Unidas para a Infância. Secretaria de Atenção a Saúde. http:// bvsms.saude.gov.br/bvs/publicacoes/violencia_criancas_ adolesc.pdf 
Bronfenbrenner, U. (2002). A ecologia do desenvolvimento humano: experimentos naturais e planejados. Editora Artes Médicas do Sul.

Caleiro, F. M., \& Silva, R. S. (2012). Técnicas de modificação do comportamento de crianças com treinamento de pais. Encontro Revista de Psicologia, 15(23),129-142. https:// revista.pgsskroton.com/index.php/renc/article/view/2475

Cecconello, A. M., Antoni, C., \& Koller, S. H. (2003). Práticas educativas, estilos parentais e abuso físico no contexto familiar. Psicologia em Estudo, 8(spe), 45-54. https://doi. org/10.1590/S1413-73722003000300007

Gaspar, T. G. S., \& Matos, M. M. M. G. (2016). Escala de Avaliação das Práticas Parentais: Controlo e Aceitação. Revista de Psicologia da Criança e do Adolescente, 7(1), 509-522. http:// revistas.lis.ulusiada.pt/index.php/rpca/article/view/2429

Germer, C. K., Siegel. R. D., \& Fulton, P. R. (2016). Mindfulness and psychotherapy. Guilford Press.

Gonçalves, J., Oliveira, A. J., \& Silva, J. V. A. (2018). Psicologia Cognitivo-Comportamental e experiência de estágio em Psicologia Clínica: Avaliação Psicológica da Ansiedade. Revista Humanas Sociais \& Aplicadas, 8(21),28-36. https:// doi.org/10.25242/887682120181344

Gonçalves, J., Oliveira, A. J., \& Delvan, J. S. (2018, outubro). Grupos de Orientação para pais no Brasil: Revisão Sistemática. In Anais do $48^{\circ}$ Reunião Anual da Sociedade Brasileira de Psicologia - SBP, São Leopoldo, RS, Brasil. http://www. sbponline.org.br/2018/10/48a-reuniao-anual-da-sbp

Goulart, V. R., Wagner, A., Barbosa, P. V., \& Mosmann, C. P. (2015). Repercussões do conflito conjugal para o ajustamento de crianças e adolescentes: Um estudo teórico. Interação em Psicologia, 19(1), 147-159. https://revistas.ufpr.br/ psicologia/article/view/35713

Hoghughi, M. (2004). Introdução à parentalidade. Sage.

Instituto Brasileiro de Geografia e Estatística. (2010). Censo Demográfico. https://www.ibge.gov.br/estatisticas/sociais/ populacao/25089-censo-1991-6.html?=\&t=o-que-e

Knapp, P., \& Beck, A. T. (2008). Fundamentos, modelos conceituais, aplicações e pesquisa da terapia cognitiva. Revista Brasileira de Psiquiatria, 30(2), 54-64. https://doi. org/10.1590/S1516-44462008000600002

Knox, M., Burkhart, K., \& Howe, T. (2011). Efeitos do programa de Criação de Riscos com Risco Seguro para Crianças sobre problemas de externalização das crianças. Relações familiares, 60(1), 491-503.
Leme, V. B. R., Del Prette, Z.A.P. D., Koller, S. H., \& Prette, A. D (2016). Habilidades sociais e o modelo bioecológico do Desenvolvimento Humano: análise e perspectivas. Revista Psicologia \& Sociedade, 28(1), 181-193. https://doi. org/10.1590/1807-03102015aop001

Macarini, S. M., Martins, G. D. F., Minetto, M. F. J., \& Vieira, M. L. (2010). Práticas parentais: uma revisão da literatura brasileira. Arquivos Brasileiros de Psicologia, 62(1), 119 134. http://pepsic.bvsalud.org/scielo.php?script=sci_ arttext\&pid=S1809-52672010000100013

Manfroi, E. C., Macarini, S. M., \& Vieira, M. L. (2011). Comportamento parental e o papel do pai no desenvolvimento infantil. Revista Brasileira de crescimento e desenvolvimento humano, 21(1), 59-69. http://pepsic.bvsalud.org/scielo.php?script=sci_ arttext\&pid=S0104-12822011000100007

Martins, C. B. G. (2010). Maus tratos contra crianças e adolescentes. Revista Brasileira de Enfermagem, 63(4), 6605. https://doi.org/10.1590/S0034-71672010000400024

Martins, G. L. L. (2014). O ambiente familiar e o desenvolvimento das funções executivas: Estudo correlacional com crianças de 3 a 6 anos. (Dissertação de Mestrado). Programa de Distúrbios do Desenvolvimento, Universidade Presbiteriana Mackenzie, São Paulo, SP, Brasil. http://tede.mackenzie.br/ jspui/handle/tede/1633

Miguel, T. P., \& Contino, A. L. B. (2013). A influência da televisão sobre o comportamento psicossocial de crianças. Revista Científica da Faminas, 9(1). http://www.faminas.edu.br/ upload/downloads/20131227154815_779651.pdf

Momo, M. (2014). As crianças de hoje não são mais como antigamente! Implicações culturais do mundo contemporâneo para os modos de ser criança e de viver a infância. Revista Textura, 16(32),1-15. http://www. periodicos.ulbra.br/index.php/txra/article/view/1243

Mondin, E. M. C. (2008). Práticas educativas parentais e seus efeitos na criação dos filhos. Psicologia Argumento, 26(54), 233-244. https://periodicos.pucpr.br/index.php/ psicologiaargumento/article/view/19885/19187

Neufeld, C. B. (2017). Aspectos técnicos e o processo em TCCG. In C. B. Neufeld, \& B. P. Rangé (Orgs.), Terapia cognitivocomportamental em grupos: das evidências à prática (Cap. 2, pp. 33-54). Artes Médicas do Sul.

Neufeld, C. B., Godoi, K. \& Propais I (2014). Tópicos CognitivoComportamentais de orientação de pais em contexto de atendimento clínico. In C. B. Neufeld (Org.), Intervenções e pesquisas em terapia cognitivo-comportamental com indivíduos e grupos (Cap. 4, pp. 116-147). Sinopsys. 
Paiva, N. M. M., \& Costa, J. S. (2015). A influência da tecnologia na infância: desenvolvimento ou ameaça? Psicologia.PT: o portal dos psicólogos. https://www.psicologia.pt/artigos/textos/ A0839.pdf

Pasquali, L., Gouveia, V. V., Santos, W. S., Fonsêca, P. N., Andrade, J. M., \& Lima, T. J. S. (2012). Questionário de Percepção dos Pais: Evidências de uma Medida de Estilos Parentais. Paidéia, 22(52), 155-164. https://doi.org/10.1590/S0103$863 \times 2012000200002$

Patias, N. D., Siqueira, A. C., \& Dias, A. C. G. (2013). Práticas educativas e intervenção com pais: a educação como proteção ao desenvolvimento dos filhos. Revista Mudanças Psicologia da Saúde, 21(1,) 29-40. http://dx.doi. org/10.15603/2176-1019/mud.v21n1p29-40

Pereira, S., Costa, R., Tojal, C., \& Tendais, I. (2018). Primeiras interações: um estudo comparativo entre mães e pais. Arquivos Brasileiros de Psicologia, 70(1), 98-109. https://bit. ly/3mKyANs

Portwood, S. G., Lambert, R. G., Abrams, L. P., \& Nelson, E. B. (2011). Uma avaliação dos adultos e crianças juntas (ACT) contra a violência dos pais aumentando o programa Kids Safe. Journal of Primary Prevention, 32(1), 147-160.

Prati, L. E., \& Koller, S. H. (2011). Relacionamento conjugal e transição para a coparentalidade: perspectiva da psicologia positiva. Psicologia Clínica, 23(1), 103-118. http://pepsic.bvsalud.org/scielo.php?script=sci_ arttext\&pid=S0103-56652011000100007

Rates, S. M. M., Melo, E. M., Mascarenhas, M. D. M., \& Malta, D. C.(2015). Violence against children: an analysis of mandatory reporting of violence, Brazil 2011. Ciência \& Saúde Coletiva, 20(3), 655-665. https://doi. org/10.1590/1413-81232015203.15242014

Rebelo, A. (2012). A Segurança dos Modelos Internos e o Conhecimento Emocional nas Crianças de Idade PréEscolar. Psicologia: Reflexão e Crítica, 3(26), 591-598. https:// doi.org/10.1590/S0102-79722013000300019
Ribeiro, E. J., Gomes, M. M., \& Felizardo, S. A. (2015). Parentalidade e estilos educativos: Perspectivas de pais e crianças (educação pré-escolar). Revista de Estudios e Investigación en Psicología y Educación, 66-68. https://doi.org/10.17979/ reipe.2015.0.05.227

Ribeiro, E. M., Eckert, E, R., Souza, A. I. J., \& Silva, A, M. F. (2007). Castigo físico adotado por pais acompanhantes no disciplinamento de crianças e adolescentes. Acta Paulista de Enfermagem, 20(3), 277-283. https://doi.org/10.1590/ S0103-21002007000300006

Silva, J. A., \& Williams, L. C. A. (2016). Um Estudo de caso com o programa parental ACT para educar crianças em ambientes seguros. Temas em Psicologia, 24(2), 743-755. http://dx.doi.org/10.9788/TP2016.2-19Pt

Teixeira, M. A. P., Bardagi, M. P., \& Gomes, W, B. (2004). Refinamento de um instrumento para avaliar responsividade e exigência parental percebidas na adolescência. Avaliação Psicológica, 3(1), 01-12. http://pepsic.bvsalud.org/scielo.php?script=sci_ arttext\&pid=S1677-04712004000100001

Weber, L. N. D., Prado, P. M., Viezzer, A. P., \& Brandenburg, O. J. (2004). Identificação de Estilos Parentais: O Ponto de Vista dos Pais e dos Filhos. Psicologia: Reflexão e Crítica, 17(3) 323-331. Doi: https://doi.org/10.1590/S010279722004000300005

Weinfurter, G. S. (2015). Violência Infantil Doméstica: forte influência no aprendizado (Especialização). Curso de Direitos Humanos, Universidade Federal do Paraná, Curitiba.

Weymouth, L. A., \& Howe, T. R. (2011). Uma avaliação multi-site do Programa de Prevenção da Violência para Crianças com Criação de Pais. Revisão dos Serviços para Crianças e Jovens, 3(1), 1960-1967. 